\title{
Catching a ride: using hypervelocity stars for interstellar and intergalactic travel
}

\begin{abstract}
Hypervelocity stars are unique among the stars in the galaxy for their extreme velocities relative to the galactic center, in some cases achieving galactic escape velocity. Dozens of hypervelocity star candidates have been identified so far. One population includes B-type stars apparently ejected from the galactic core. A second population has been identified within the plane of the galaxy with no single origin. As a fast-moving energy source, hypervelocity stars could be uniquely valuable property in the galaxy for advanced civilizations. Given their potential for transportation and exploration across the plane of the galaxy or to other galaxies, they could serve as prime objects for Search for Extraterrestrial Intelligence (SETI) searches or as longduration transports for far-future human exploratory missions. In addition, some hypervelocity stars may be entering our galaxy from extragalactic sources, making them possible mechanisms by which intelligences in neighboring galaxies could be exploring ours.
\end{abstract}

Keywords: SETI, hypervelocity stars, intergalactic travel, three-body interactions, chaotic interactions
Volume 2 Issue 3 - 2018

Joseph L Breeden

Prescient Models LLC, USA

Correspondence: Joseph L Breeden, Prescient Models LLC, 1600 Lena St., Suite E3, Santa Fe, NM 87505, USA, Email breeden@prescientmodels.com

Received: April 12, 2018| Published: May 16,2018

\section{Introduction}

Traveling to another star always involves accelerating enough mass to provide habitat, energy, and resources for those who would make the journey. Previous work ${ }^{1,2}$ looked at how chaotic orbits of a binary asteroid passing near our sun might in very rare occasions provide all of this "for free". A similar process may be operating continuously in the galactic core and around other black holes whereby binary stars interact with the central black hole. Via a chaotic trajectory, one of those stars may be accelerated to extreme velocity. As a massive, high velocity energy source, might these hypervelocity stars (HVS) serve as a galactic transport system? Most research into hypervelocity stars (HVSs) focuses on studying their properties in order to learn more about the properties of the galactic core. However, these objects should be very interesting to those interested in the search for extraterrestrial intelligence (SETI). No known HVS are near our solar system, gravitationally speaking, which is a very good thing for the stability of our system. Consequently, we also cannot consider them as a useful means of interstellar or intergalactic travel until we have achieved the ability to travel hundreds of light-years from our home system. Nevertheless, other more advanced species may have the ability to use HSVs as a means of travel. In the following sections we will discuss the properties of HVSs, their suitability for interstellar or intergalactic travel, and their feasibility as planetary and SETI search targets. Hypervelocity stars (HVS) were first predicted by Hills $^{3}$ and then discovered by Brown et al. ${ }^{4}$ The pace of discovery of HVS candidates has increased in recent years. ${ }^{5-11}$ Their name comes from the fact that these stars are traveling at or near galactic escape velocity with the most extreme cases exceeding $1000 \mathrm{~km} / \mathrm{s}$. Recent surveys have shown a surplus of such stars with velocities greater than $350 \mathrm{~km} / \mathrm{s}$ in the stellar velocity distribution for our galaxy, ${ }^{12}$ suggesting a different mechanism for attaining velocity from the rest of the stellar distribution.

\section{HSV properties}

For those stars apparently originating from the galactic core, Hills ${ }^{3}$ proposed model of a 3-body interaction is the leading candidate. In this model, a binary star has a close interaction with the central black hole. One star is captured or consumed while the other is ejected at high velocity. If the galactic core houses a binary black hole, then single stars could be ejected at extreme velocities as well. ${ }^{13}$ Overwhelming evidence exists for a super-massive black hole (MBH) in the Galactic center. ${ }^{14,15}$ To date, all candidate HVSs originating from the galactic core are B-type stars. The binary star-black hole interaction appears to be 5.5 times more efficient at ejecting B-type stars than low-mass $\mathrm{F}$ and $\mathrm{G}$ stars. ${ }^{16}$ The average ejection rate is estimated to be between 0.6 B-type HVS per million years ${ }^{6}$ and 1.5 per million years. ${ }^{5}$ At least 40 such candidates have been found to date $\mathrm{e}^{5,10,11}$ at 50 to $120 \mathrm{kiloparsec}$ $(\mathrm{kpc})$ distances. Given this ejection rate and average life of these stars of 1.6 to $3.5 \times 10^{8} \mathrm{yr}$, between 100 and 600 B-type HVS should exist in the galaxy at any time, assuming steady production over multiple B-star lifetimes. Semi-analytical models predict that there may be roughly 100 HVSs within $8 \mathrm{kpc}$ of the Galactic center due to the breakup of equal-mass binaries. ${ }^{13,17}$

More recent surveys have found another 32 candidate low-mass HVS of G and K types. ${ }^{7,9}$ Although these HVS are not yet confirmed, theoretical models suggest plausible mechanisms for creating such HSV. None of these stars appear to originate from the galactic center. Within the galactic plane, proposed origins include binary black hole -single star interactions. ${ }^{18}$ One small group of HVS may be stripped stars from a passing dwarf galaxy. ${ }^{19}$ Globular star clusters are another possible source of HVS, since globular star clusters undergoing core collapse $^{20,21}$ are known to have a comparatively high rate of 3-body interactions. Any ejected stars would be older, smaller Population II stars; no O or B stars. However, since globular clusters have no preferential alignment to the galactic plane, ejected stars would exit with random trajectories and therefore predominantly in the galactic halo, a distribution similar to the B-type HVS being ejected from the galactic core. We also have evidence of HVS being ejected by other galaxies. An entire globular cluster has been ejected from M87 with radial velocity is $-1026 \pm 13 \mathrm{~km} / \mathrm{s}$ and velocity relative 
to M87 of $2300 \mathrm{~km} / \mathrm{s}^{22}$ Simulations suggest that over $1000 \mathrm{HVSs}$ may be approaching the Milky Way due to ejection from M31 via the disruption of stellar binaries by the galactocentric MBH or the ejection of stars by an in-spiraling intermediate-mass black hole. ${ }^{23}$

The three-body problem has been known since the work of Henri Poincar in the 1880 s to defy simple solutions. Work of the last few decades, mostly via computer simulation, has established the gravitational three-body problem as one of the simplest examples of chaos theory. One of the defining features of chaos theory is the rapid growth of small perturbations so that nearby trajectories diverge exponentially. For this reason, computer simulations of chaotic systems must be extremely high fidelity and are computationally intensive. Star-star interactions are elastic, except in cases where the impact parameter is less than a few stellar radii. However, binary star-single star interactions are inelastic, allowing for energy transfer and exchange interactions. ${ }^{24,25}$ Such 3-body interactions have been proposed as the mechanism for gravothermal oscillations of globular star clusters, ${ }^{26}$ the capture of Triton by Neptune, ${ }^{27}$ and as a general mechanism for capturing irregular moons around gas giant planets. ${ }^{28}$ If chaotic interactions are the mechanism for creating HSV, it will have implications for the presence and habitability of any planetary systems about those objects. We do not know at this time if these stars have any accompanying planets, but simulations suggest that planetary systems like those of our sun are unlikely to survive chaotic interactions. ${ }^{29}$ However, a binary star system is likely to have only tightly bound planets. These could be Jupiter-mass planets, as several such planets with orbital period's $\leq 1$ day have been identified. ${ }^{30,31}$ Therefore, specific studies of binary star-black hole interactions have been performed ${ }^{32}$ where each star has tightly bound massive planets. Depending upon initial orbital conditions, those studies suggest a probability of $30 \%$ to $70 \%$ for HSV to retain tightly bound planets. Further, there is a high probability for the creation of hypervelocity planets (HVP) from the captured star.

\section{Interstellar travel with HVS}

Although scientifically interesting, HVS take on special importance in a galaxy with advanced civilizations. As a powerful energy source moving at high velocity through the galaxy, they are valuable real estate. For exploration or transportation, they present an opportunity to move whole civilizations at comparatively low cost. Most of the HVS discovered so far are in the galactic halo, because our view of them is not obscured by dust. The most valuable HVS would seem to be those traveling in or near the galactic plane. If the observed and theoretically proposed anisotropy of HVS ejected by the galactic core persists, ${ }^{5,33}$ the probability of finding an HVS in the galactic plane would be quite low. If any of the currently identified B-type HVS have been used for transport by advanced civilizations, that role must have been completed long ago given their current distance from the galactic plane. The recently proposed population of $\mathrm{G}$ and $\mathrm{K}$ type stars is potentially more interesting. Recent discoveries from the Sloan SEGUE survey, however, have identified $20 \mathrm{G}$ and $\mathrm{K}$ type dwarf stars as candidate hypervelocity stars, some with velocities exceeding $1000 \mathrm{~km} / \mathrm{s}$ relative to the galactic center and computed to be on escape trajectories. ${ }^{9}$

These stars are projected to be created within the galactic plane and may prove more useful to an advanced civilization. These lowmass HVS present a unique opportunity for intergalactic travel. A 0.5 solar mass star would survive as a main sequence dwarf for roughly $100 \mathrm{Gyr}$. The time needed to reach Andromeda at a distance of
$2.5 \times 10^{6} \mathrm{LY}$, a $1000 \mathrm{~km} / \mathrm{s} \mathrm{HVS}$ would arrive in $760 \mathrm{Myr}-w e l l$ within the star's lifetime. Therefore, although the previously discovered B type hypervelocity stars were unsuitable for the journey, riding along with a dwarf star is quite feasible. We do not yet have sufficiently precise trajectory simulations for the identified G and K type HVS to know if any are headed to nearby galaxies. Once determined, those would have to be considered extremely interesting objects. Intriguingly, calculations suggest that over 1000HVS may be approaching the Milky Way from the Andromeda Galaxy (M31). ${ }^{23}$ If a civilization in that galaxy wanted to reach our own, this would appear to be an ideal means of transport. If found, those stars would be extremely interesting targets of study.

\section{Capture and habitability}

For an external species to use an HVS for transportation, the population sent to the HVS must execute a capture maneuver. Since the HVS is a high velocity energy source, orbital insertion does not require carrying fuel. Various kinds of solar power or solar sail technologies could be employed. The presence of residual planetesimals also allow for dynamical capture. Using HVS as long-duration transportation implies that the system must in some sense be habitable. ${ }^{34}$ The question can be viewed in two ways. Could a pre-ejection civilization survive ejection or could a post-ejection civilization be established? (Table 1) (Table 2)

Table I Hypervelocity star candidates as summarized by aBrown et al., ${ }^{5}$

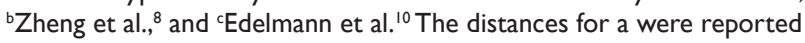
relative to the galactice center, so the uncertainties are an additional $\pm 8.3 \mathrm{kpc}$ from the Sun added to what is listed. The remaining distances were heliocentric.

\begin{tabular}{llll}
\hline $\mathbf{V}_{\mathbf{G C}}$ & $\mathbf{d}[\mathbf{k p c}]$ & Mass & Catalog \\
\hline 673.1 & $107 \pm 15$ & $3.23 \pm 0.13$ & $\mathrm{~J} 090744.99+024506.89^{\mathrm{a}}$ \\
551.5 & $70 \pm 10$ & $4.24 \pm 0.16$ & $\mathrm{~J} 091301.01+305119.83^{\mathrm{a}}$ \\
650.1 & $50 \pm 5$ & $3.58 \pm 0.11$ & $\mathrm{~J} 091759.47+672238.35^{\mathrm{a}}$ \\
501.4 & $58 \pm 7$ & $3.06 \pm 0.11$ & $\mathrm{~J} 110557.45+093439.47^{\mathrm{a}}$ \\
402 & $53 \pm 6$ & $3.76 \pm 0.12$ & $\mathrm{~J} 113312.12+010824.87^{\mathrm{a}}$ \\
408.3 & $58 \pm 10$ & $3.42 \pm 0.20$ & $\mathrm{~J} 094214.03+200322.07^{\mathrm{a}}$ \\
458.8 & $77 \pm 12$ & $3.54 \pm 0.16$ & $\mathrm{~J} 102137.08005234 .77^{\mathrm{a}}$ \\
416.7 & $53 \pm 6$ & $2.65 \pm 0.11$ & $\mathrm{~J} 120337.85+180250.35^{\mathrm{a}}$ \\
416.5 & $67 \pm 8$ & $2.73 \pm 0.14$ & $\mathrm{~J} 105009.59+031550.67^{\mathrm{a}}$ \\
423.9 & $107 \pm 19$ & $3.05 \pm 0.18$ & $\mathrm{~J} 105248.30000133 .94^{\mathrm{a}}$ \\
409.4 & $105 \pm 16$ & $3.18 \pm 0.15$ & $\mathrm{~J} 104401.75+061139.02^{\mathrm{a}}$ \\
328.3 & $67 \pm 10$ & $2.99 \pm 0.12$ & $\mathrm{~J} 113341.09012114 .25^{\mathrm{a}}$ \\
346.2 & $71 \pm 12$ & $2.85 \pm 0.15$ & $\mathrm{~J} 122523.40+052233.84^{\mathrm{a}}$ \\
435.8 & $49 \pm 4$ & $3.91 \pm 0.09$ & $\mathrm{~J} 164156.39+472346.12^{\mathrm{a}}$ \\
446.2 & $80 \pm 11$ & $3.27 \pm 0.12$ & $\mathrm{~J} 232904.94+330011.47^{\mathrm{a}}$ \\
492 & $98 \pm 15$ & $3.12 \pm 0.17$ & $\mathrm{~J} 113517.75+080201.49^{\mathrm{a}}$ \\
396.6 & $76 \pm 11$ & $2.79 \pm 0.12$ & $\mathrm{~J} 113637.13+033106.84^{\mathrm{a}}$ \\
391.9 & $113 \pm 21$ & $3.70 \pm 0.21$ & $\mathrm{~J} 103418.25+481134.57^{\mathrm{a}}$ \\
488.7 & $85 \pm 13$ & $2.66 \pm 0.16$ & $\mathrm{~J} 114146.44+044217.29^{\mathrm{a}}$ \\
423.2 & $112 \pm 20$ & $3.04 \pm 0.16$ & $\mathrm{~J} 215629.01+005444.18^{\mathrm{a}}$ \\
357.6 & $56 \pm 7$ & $2.93 \pm 0.12$ & $\mathrm{~J} 111136.44+005856.44^{\mathrm{a}}$ \\
\hline & & &
\end{tabular}


Table Continued

\begin{tabular}{llll}
\hline $\mathbf{V}_{\mathbf{G C}}$ & $\mathbf{d}[\mathbf{k p c}]$ & Mass & Catalog \\
\hline 308.6 & $45 \pm 9$ & $3.90 \pm 0.31$ & $\mathrm{~J} 002810.33+215809.66^{\mathrm{a}}$ \\
302.1 & $39 \pm 9$ & $3.00 \pm 0.33$ & $\mathrm{~J} 005956.06+313439.29^{\mathrm{a}}$ \\
282.8 & $83 \pm 16$ & $3.61 \pm 0.25$ & $\mathrm{~J} 074950.24+243841.16^{\mathrm{a}}$ \\
279.9 & $31 \pm 4$ & $2.84 \pm 0.18$ & $\mathrm{~J} 081828.07+570922.07^{\mathrm{a}}$ \\
275.2 & $40 \pm 5$ & $2.75 \pm 0.16$ & $\mathrm{~J} 090710.07+365957.54^{\mathrm{a}}$ \\
315.2 & $54 \pm 6$ & $2.30 \pm 0.12$ & $\mathrm{~J} 095906.47+000853.41^{\mathrm{a}}$ \\
283.9 & $65 \pm 11$ & $2.31 \pm 0.21$ & $\mathrm{~J} 101359.79+563111.65^{\mathrm{a}}$ \\
347.1 & $45 \pm 7$ & $2.80 \pm 0.16$ & $\mathrm{~J} 103357.26011507 .35^{\mathrm{a}}$ \\
294.1 & $74 \pm 16$ & $2.95 \pm 0.26$ & $\mathrm{~J} 104318.29013502 .51^{\mathrm{a}}$ \\
319.6 & $36 \pm 6$ & $3.26 \pm 0.20$ & $\mathrm{~J} 112255.77094734 .92^{\mathrm{a}}$ \\
288.9 & $31 \pm 5$ & $2.47 \pm 0.19$ & $\mathrm{~J} 115245.91021116 .21^{\mathrm{a}}$ \\
289.1 & $43 \pm 8$ & $3.56 \pm 0.19$ & $\mathrm{~J} 140432.38+352258.41^{\mathrm{a}}$ \\
286.8 & $24 \pm 4$ & $2.15 \pm 0.16$ & $\mathrm{~J} 141723.34+101245.74^{\mathrm{a}}$ \\
283.9 & $61 \pm 14$ & $3.21 \pm 0.24$ & $\mathrm{~J} 154806.92+093423.93^{\mathrm{a}}$ \\
288.3 & $38 \pm 8$ & $3.29 \pm 0.25$ & $\mathrm{~J} 180050.86+482424.63^{\mathrm{a}}$ \\
277.8 & $32 \pm 7$ & $3.21 \pm 0.26$ & $\mathrm{~J} 232229.47+043651.45^{\mathrm{a}}$ \\
$477 \pm 10$ & $9.1 \pm 0.7$ & $13.4 \pm 2.2$ & $\mathrm{~J} 091206.52+091621.8^{\mathrm{b}}$ \\
563 & 8 & $61 \pm 12$ & $\mathrm{H} 004375439^{\mathrm{c}}$ \\
\hline
\end{tabular}

Table 2 Hypervelocity star candidates as summarized by dPalladino et al., ${ }^{9} \mathrm{~L}$ et al., ${ }^{7}$ Kollmeier et al., ${ }^{6}$ and 8 Hirsh et al." Heliocentric distances are shown.

\begin{tabular}{lll}
\hline $\mathbf{V}_{\mathbf{r f}}$ & $\mathbf{d}[\mathbf{k p c}]$ & Catalog \\
\hline 802.2 & 3.7 & $\mathrm{~J} 60306.77+825829.1^{\mathrm{d}}$ \\
628.6 & 5.68 & $\mathrm{~J} 23433.42+262327.5^{\mathrm{d}}$ \\
641.8 & 4.06 & $\mathrm{~J} 160620.65+42451.5^{\mathrm{d}}$ \\
1086.8 & 3.19 & $\mathrm{~J} 185018.09+191236.1^{\mathrm{d}}$ \\
916.3 & 3.13 & $\mathrm{~J} 35429.2761354 .1^{\mathrm{d}}$ \\
793.9 & 3.06 & $\mathrm{~J} 64337.13+291410^{\mathrm{d}}$ \\
769.1 & 2.48 & $\mathrm{~J} 202446.41+121813.4^{\mathrm{d}}$ \\
937.3 & 3.31 & $\mathrm{~J} 11933.45+384913^{\mathrm{d}}$ \\
992.9 & 3.82 & $\mathrm{~J} 172630.6+75544^{\mathrm{d}}$ \\
712.9 & 3.7 & $\mathrm{~J} 73542.35+164941.4^{\mathrm{d}}$ \\
731.4 & 3.14 & $\mathrm{~J} 25450.18+333158.4^{\mathrm{d}}$ \\
715.7 & 2.91 & $\mathrm{~J} 134427.8+282502.7^{\mathrm{d}}$ \\
840.7 & 4.6 & $\mathrm{~J} 225912.13+74356.5^{\mathrm{d}}$ \\
649.8 & 2.22 & $\mathrm{~J} 95816.39+5224.4^{\mathrm{d}}$ \\
672.8 & 3.26 & $\mathrm{~J} 74728.84+185520.4^{\mathrm{d}}$ \\
601.4 & 1.78 & $\mathrm{~J} 64257.02+371604.2^{\mathrm{d}}$ \\
649.1 & 4.35 & $\mathrm{~J} 165956.02+392414.9^{\mathrm{d}}$ \\
622.7 & 4.56 & $\mathrm{~J} 110815.19155210 .3^{\mathrm{d}}$ \\
606.7 & 5.88 & $\mathrm{~J} 145132.12+3258^{\mathrm{d}}$ \\
$-288 \pm 5$ & $4.1 \pm 0.3$ & $\mathrm{~J} 221853.48004030 .9^{\mathrm{e}}$ \\
\hline
\end{tabular}

\begin{tabular}{lll}
$-354 \pm 9$ & $6.3 \pm 1.2$ & $\mathrm{~J} 221625.92+003555.4^{\mathrm{e}}$ \\
\hline $\mathbf{V}_{\mathbf{r f}}$ & $\mathbf{d}[\mathbf{k p c}]$ & Catalog \\
\hline $317 \pm 8$ & $8.1 \pm 0.7$ & $\mathrm{~J} 222620.74+004135.6^{\mathrm{e}}$ \\
$-315 \pm 5$ & 3.30 .4 & $\mathrm{~J} 172915.20+431717.2^{\mathrm{e}}$ \\
$382 \pm 8$ & $7.1 \pm 0.6$ & $\mathrm{~J} 125210.66+300147.9^{\mathrm{e}}$ \\
$313 \pm 8$ & $6.7 \pm 0.4$ & $\mathrm{~J} 074817.52+360034.4^{\mathrm{e}}$ \\
$359 \pm 7$ & $6.0 \pm 0.7$ & $\mathrm{~J} 123237.34+165526.9^{\mathrm{e}}$ \\
$373 \pm 5$ & $5.6 \pm 0.5$ & $\mathrm{~J} 121112.12+402029.5^{\mathrm{e}}$ \\
$-392 \pm 18$ & $10 \pm 0.9$ & $\mathrm{~J} 172733.94+322459.1^{\mathrm{e}}$ \\
$384 \pm 8$ & $8.5 \pm 0.9$ & $\mathrm{~J} 171826.16+645745.8^{\mathrm{e}}$ \\
$371 \pm 8$ & $4.3 \pm 0.3$ & $\mathrm{~J} 124814.84+284010.2^{\mathrm{e}}$ \\
$-295 \pm 4$ & $3.1 \pm 0.3$ & $\mathrm{~J} 160912.29+345706.8^{\mathrm{e}}$ \\
$-297 \pm 4$ & $6.5 \pm 0.4$ & $\mathrm{~J} 203328.51+142607.0^{\mathrm{e}}$ \\
$-412.3 \pm 5.5$ & & $\mathrm{~J} 061118.63+642618.5^{\mathrm{f}}$ \\
$-409.0 \pm 5.9$ & & $\mathrm{~J} 074557.31+181246.7^{\mathrm{f}}$ \\
$-407.1 \pm 16.3$ & & $\mathrm{~J} 224052.56+011332.1^{\mathrm{f}}$ \\
$-404.4 \pm 17.2$ & & $\mathrm{~J} 211321.02+103456.7^{\mathrm{f}}$ \\
$425.4 \pm 3.1$ & & $\mathrm{~J} 111107.85+585357.2^{\mathrm{f}}$ \\
$401.8 \pm 13.1$ & & $\mathrm{~J} 224740.09-004451.6^{\mathrm{f}}$ \\
751 & 19 & $\mathrm{US} 7088^{\mathrm{g}}$ \\
\hline
\end{tabular}

The first question is probably simple to answer. The habitable zone in a planetary system as defined by the possible presence of liquid water would not extend as close to a star as the highly bound planets hypothesized to survive ejection. Simulations have not been conducted on all possible planetary systems, so it is possible that a binary star system could have planets in the habitable zone, but even if those planets survived ejection, the environment extremes experience during the chaotic trajectories experienced would be so great as to make it unlikely that any but the most basic and hardened life would survive. The possibility of inhabiting an HVS system post-ejection seems more promising. Although habitable planets are unlikely to survive ejection, that does not seem to be an important criteria for a useful HVS system. Even a planet with a biosphere is more likely to be inhospitable to an incoming species. Instead, all that is needed is raw material. Any orbital debris, such as asteroids or comets, would provide raw material for establishing artificial habitats. Solar power and the star's gravity provide energy and transportation. The presence or absence of planets is largely irrelevant, but the possibility that planets could survive suggests that some raw material will be present. Of course, the easiest transport method would be to send either a robotic or organic seed population that would utilize the energy and orbital debris to sustain operations for a long journey. The HVS would serve as a probe relaying observations back to the progenitor civilization.

\section{SETI and HVS}

The nearest candidate HVS is 1kiloparsec (kpc), or approximately 3,200 light years (LY) away. For comparison, the galactic center is $27.2 \times 10^{6} \pm 1.1 \mathrm{LY}(8.34 \pm 0.34 \mathrm{kpc})$ away. ${ }^{35}$ Given that and the obvious technical challenges, human civilization will not be catching a ride on a hypervelocity star any time soon. Instead, we need to consider HVS 
as resources to other advanced civilizations. Given the obvious value of these objects, HVS may be one of the best possible SETI targets. Currently we can detect signals up to $300 \mathrm{pc},{ }^{36}$ roughly 1,000 lightyears using a Keck-class telescope. That puts the nearest HVS about $3 \mathrm{x}$ too far away for current means, but this is close enough that we may soon be able to try. Given the small number of HVS candidates, adding them as targets now for any SETI search makes good sense. Table 1 \& Table 2 capture most of the top HVS candidates for inclusion in such studies. VGC is the velocity relative to the galactic center. $\mathrm{d}$ is the distance from the Sun. The mass reported in Table 1 is in solar masses. Objects J221853.48004030.9 and J221625.92+003555.4 from Table 2 are particularly interesting, as Li et al. ${ }^{7}$ suggest that these have originated in M31 or M32 as hypothesized by Sherwin et al. ${ }^{23}$ With known technology and physical laws, intergalactic HVS may be the best possible means of crossing the gap between galaxies, so we should definitely be monitoring their approach to see what they might bring.

\section{Conclusion}

Although many suggest statistically that many advanced civilizations should exist in our galaxy, finding them may not be simple. As our own civilization has become more advanced, our communications have become more energy efficient and narrow band. Of course, if someone wanted to contact us, one assumes that they could do so relatively easily with a narrow beam transmission pointed our direction. The more challenging problem is finding civilizations that are not trying to contact us. That will require deep searches of high-probability objects. In the search of ideal candidates, HVS are not where one would look to find indigenous life. Any locally evolved civilization is unlikely to survive any of the proposed ejection mechanisms. However, post-ejection, HVS should be among the most valuable properties in the galaxy for an advanced civilization. So much so that if the galaxy hosts multiple competing civilizations, these could be a cause for conflict. In the realm of signal detection, conflict is a prime opportunity to pick up stray signals. Because of all the properties discussed here, hypervelocity stars should be near the top of SETI observation lists. Proposals to search for planets orbiting $\mathrm{HVS}^{37}$ are a good start for studying such systems, even if the presence of planets is not essential for the use of HVS for transport by sentient species. The tables provided here collect 77 high value candidates identified so far. Although more will be found, theories suggest that we may have already found as much as one third of all such objects.

\section{Funding details}

This research was sponsored by Prescient Models LLC, Santa Fe, NM, USA.

\section{Acknowledgements}

The author wishes to thank Prescient Models LLC for its support of this research.

\section{Conflict of interest}

The author declares that there is no conflict of interest.

\section{References}

1. Breeden JL. Gravitational assist via near-sun chaotic trajectories of binary objects. Journal of the British Interplanetary Society. 2013;66:190-194.

2. Breeden JL. SETI implications of gravitational assist via chaotic trajectories of binary objects. Acta Astronautica. 2014;93:106-111.

3. Hills JG. Hyper-velocity and tidal stars from binaries disrupted by a massive galactic black hole. Nature. 1988;331:687-689.

4. Brown WR, Geller MJ, Kenyon SJ, et al. Discovery of an unbound hypervelocity star in the Milky Way halo. The Astrophysical Journal. 2005;622(1):L33-L36.

5. Brown WR, Geller MJ, Kenyon SJ. Mmt hypervelocity star survey. iii. the complete survey. The Astrophysical Journal. 2014;787(1):89.

6. Kollmeier JA, Gould A, Knapp G, et al. Old-population hypervelocity stars from the galactic center: Limits from the sloan digital sky survey. The Astrophysical Journal. 2009;697(2):1543-1548.

7. Li Y, Luo A, Zhao G, et al. Metal-poor hypervelocity star candidates from the sloan digital sky survey. The Astrophysical Journal Letters. 2012;744(2):L24-L29.

8. Zheng Z, Carlin JL, Beers TC, et al. The first hypervelocity star from the Lamost survey. The Astrophysical Journal Letters. 2014;785(2):L23.

9. Palladino LE, Schlesinger KJ, Bockelmann KH, et al. Hypervelocity star candidates in the Segue G \& K dwarf sample. The Astrophysical Journal. 2014;780(1):57.

10. Edelmann H, Napiwotzki R, Heber U, et al. He 0437-5439: An unbound hypervelocity main-sequence b-type star. The Astrophysical Journal Letters. 2005;634(2):L181.

11. Heber U, Toole SJO, Hirsch HA, et al. Us 708 an unbound hyper-velocity subluminous o star. Astronomy \& Astrophysics. 2005;444:1-4.

12. Brown WR, Geller MJ, Kenyon SJ. Mmt hypervelocity star survey. ii. Five new unbound stars. The Astrophysical Journal. 2012;751(1):55.

13. Yu Q, Tremaine S. Ejection of hypervelocity stars by the (binary) black hole in the galactic center. The Astrophysical Journal. 2003;599(2):1129-1138.

14. Ghez AM, Salim S, Weinberg NN, et al. Measuring distance and properties of the Milky Way's central super massive black hole with stellar orbits. The Astrophysical Journal. 2008;689(2):1044.

15. Gillessen S, Eisenhauer F, Trippe S, et al. Monitoring stellar orbits around the massive black hole in the galactic center. The Astrophysical Journal. 2009;692(2):1075.

16. Kollmeier JA, Gould A, Knapp G, et al. Segue-2 limits on metal-rich old-population hypervelocity stars in the galactic halo. The Astrophysical Journal. 2012;723(1):812-817.

17. Gould A, Quillen AC. Sagittarius a* companion S0-2: A probe of very high mass star formation. The Astrophysical Journal. 2003;592(2):935.

18. Sesana A, Haardt F, Madau P. Interaction of massive black hole binaries with their stellar environment. i. Ejection of hypervelocity stars. The Astrophysical Journal. 2006;651(1):507-596.

19. Mario GA, Julio FN, Matthias S. An alternative origin for hypervelocity stars. The Astrophysical Journal. 2009;691:L63-L66.

20. Breeden JL, Cohn HN, Hut P. The onset of gravothermal oscillations in globular cluster evolution. Astrophysical Journal. 1994;421(1):195-205.

21. Breeden JL, Cohn HN. Chaos in core oscillations of globular clusters. 
Astrophysical Journal. 1995;448(2):672-682.

22. Nelson C, Strader J, Romanowsky AJ, et al. A globular cluster toward m87 with a radial velocity $<1000 \mathrm{kms} 1$ : The first hypervelocity cluster. Technical report; 2014

23. Sherwin BD, Loeb A, O'Leary RM. Hypervelocity stars from the andromeda galaxy. Monthly Notices of the Royal Astronomical Society. 2008;386(3):1179-1191.

24. Douglas C Heggie. Binary evolution in stellar dynamics. Monthly Notices of the Royal Astronomical Society. 1975;173(3):729-787.

25. Hut P, Bachall JN. Binary-single star scattering. i-numerical experiments for equal masses. The Astrophysical Journal. 1983;268(1):319-341.

26. Lyman Spitzer. Dynamical evolution of globular clusters. USA: Princeton University Press; 1987. 192 p.

27. Agnor CB, Hamilton DP. Neptune's capture of its moon triton in a binaryplanet gravitational encounter. Nature. 2006;441(7090):192-194.

28. Darren M Williams. Forming big moons through capture around gas giant planets. Bulletin of the American Astronomical Society. 2010;42:1070.

29. Marzari F, Barbieri M. Planet dispersal in binary systems during transient multiple star phases. Astronomy \& Astrophysics. 2007;472(2):643-647.
30. Hebb L, Collier-Cameron A, Triaud AHMJ, et al. Wasp-19b: The shortest period transiting exoplanet yet discovered. The Astrophysical Journal. 2010;708(1):224.

31. Hellier C, Anderson DR, Collier CameronA, et al. Wasp-43b: the closestorbiting hot jupiter. Astronomy \& Astrophysics. 2011;535:1-5.

32. Ginsburg I, Loeb A, Wegner GA. Hypervelocity planets and transits around hypervelocity stars. Monthly Notices of the Royal Astronomical Society. 2012;423(1):948-954.

33. Lu Y, Zhang F, Yu Q. On the spatial distribution and the origin of hypervelocity stars. The Astrophysical Journal. 2010;709(2):1356-1361.

34. Lammer H, Bredehoft JH, Coustenis A, et al. What makes a planet habitable? Astronomy \& Astrophysics Review. 2009;17(2):181-249.

35. Gillessen S, Eisenhauer F, Trippe S, et al. Monitoring stellar orbits around the massive black hole in the galactic center. The Astrophysical Journal. 2009;692(2):1075.

36. Howard A, Horowitz P, Coldwell C, et al. An All-sky optical Seti Survey. USA: In Proceedings $54^{\text {th }}$ International Astronautical Congress; 2003. 8 p.

37. Fragione G, Ginsburg I. Transit probabilities around hypervelocity and runaway stars. Monthly Notices of the Royal Astronomical Society. 2017;466(2):1805-1813. 